\title{
A public health framework for the equitable global allocation of vaccines: COVID-NEEDS
}

\author{
Vageesh Jain $^{1}$ (D) Paula Lorgelly ${ }^{2}$
}

Accepted: 29 November 2021 / Published online: 13 January 2022

(C) The Author(s), under exclusive licence to Springer Nature Limited 2021

\begin{abstract}
The equitable global allocation of COVID-19 vaccines has received much attention yet been poorly defined. Understanding equity requires assessing needs for vaccines across countries. Making distinctions is especially challenging when countries perform similarly on traditional epidemiological metrics. This Viewpoint offers a novel conceptual framework (COVID-NEEDS) based on empirical evidence and public health guidance. It encompasses health, social, and economic impacts of COVID19 and associated non-pharmaceutical interventions. We intend this framework to complement existing needs assessment methods to help identify countries most in need of vaccines. We present factors to consider, but future work will be required to understand how to weight the factors and to determine the practical utility of the framework for supplementing existing COVID-19 vaccine allocation mechanisms.
\end{abstract}

Keywords Health policy $\cdot$ Public health $\cdot$ Health equity $\cdot$ Immunization $\cdot$ COVID-19

\section{Equitable allocation of COVID-19 vaccines}

Equity goes beyond equality to represent the notion that resources should be distributed based on need. Vaccine allocation should be prioritized for those with the greatest needs, and to ensure that those with similar needs have comparable access. The COVID-19 pandemic has affected populations differently, in terms of direct health risks and the impact of non-pharmaceutical interventions (NPIs) such as lockdowns. Assessing relative needs for vaccines across nations is therefore challenging. Based on supply and manufacturing constraints, The Wellcome Trust previously estimated that not everyone who needs a vaccine globally will receive one until at least 2023 [1] — notwithstanding the risk of future mutations and the potential need for novel

Vageesh Jain

vageesh.jain@ucl.ac.uk

1 Institute for Global Health, University College London, London WC1E 6BT, UK

2 Department of Applied Health Research, Institute of Epidemiology \& Health Care, University College London, London WC1E 6BT, UK 
vaccines and re-immunization. Equitable global allocation relies on an effective and evidence-based priority-setting process to compare needs across countries fairly. International decision-making about the distribution of vaccines globally (chiefly under the remit of the WHO, Gavi and Coalition for Epidemic Preparedness Innovations (CEPI) co-led partnership, COVAX) should be based on need and timely data. To date there is no consensus on a comprehensive, evidence-based, global framework to facilitate this crucial task of synthesizing relevant information.

This Viewpoint sets out a framework of factors to aid decision-makers in comparing vaccine needs between countries. The COVID-19 pandemic has had far-reaching impacts of across societies and at various points in time different populations have yielded similar results using traditional disease burden metrics such as case numbers [2] or reproduction numbers [3] (the pace of change in disease spread across a population). Plans currently in use provide valuable but limited means for assessing vaccine needs across countries. Expanding the range of factors considered, when needs appear to be very similar across countries based on current methods, can enable a more equitable pandemic response.

\section{Existing vaccine allocation plans}

The COVAX Facility was set up to facilitate equitable access to vaccines worldwide. COVAX aims to have allocated 2 billion doses, primarily for low- and middleincome countries (LMICs), by the end of 2021. The COVAX system includes two phases (Fig. 1) [4]. In the first, countries receive vaccine doses proportional to the size of their population, to vaccinate up to $20 \%$ of citizens. Once countries reach this threshold, in phase two, COVAX distributes doses to countries based on need. Parameters used to assess need include the effective reproduction number ( $\mathrm{R}$ number) and its trend, hemisphere location, universal health coverage (UHC) service

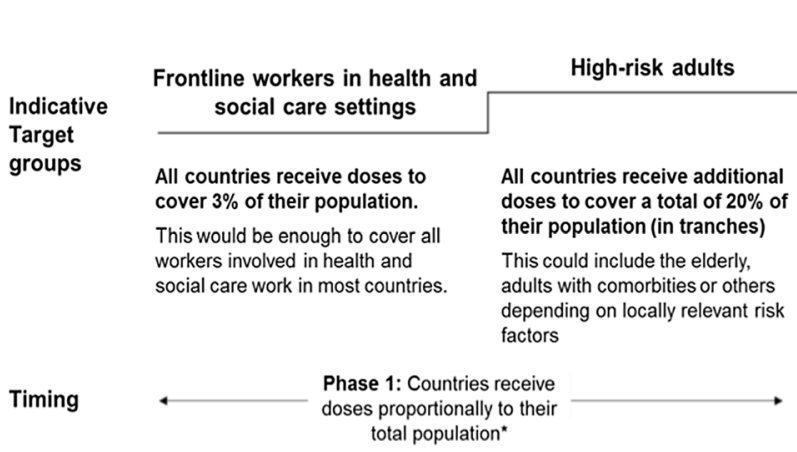

Further priority groups

A buffer will also be set aside for humanitarian deployment

Fig. 1 COVAX allocation plan overview (WHO)

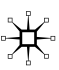


coverage index, health system saturation, and groups at a high-risk of severe disease or death [5]. Each country is scored based on the weighted averages of these parameters, accompanied by a qualitative assessment of country context. In addition to the vaccine allocations in phases one and two, COVAX reserves up to $5 \%$ of vaccine doses as part of a humanitarian buffer for populations such as refugees or asylum seekers [6].

Given global heterogeneity in population demographics and disease profiles, healthcare staffing, and vaccine acceptance, in some countries, providing vaccines for $20 \%$ of the population may suffice to cover high-risk groups, but in other countries it may not. Across high-income country populations, 18\% are aged 65 and above, compared to just 3\% in low-income countries [7]. There are also more than ten times as many physicians per capita in high-income countries [8]. Although factors like these vary globally, where there are similarities between countries, COVAX decisions can be aided by consideration of a wider range of factors, as we propose in the COVID-NEEDS framework.

A model by researchers at Vanderbilt University suggests distributing vaccines to countries based on their ability to distribute vaccines, to provide care, and whether they have helped test and develop new interventions [9]. The Fair Priority Model is another framework for equitable international allocation of COVID-19 vaccines [10]. Vaccine allocation is based on three sequential objectives or phases. Phase one uses standard expected years of life lost to calculate optimal distribution of vaccines. Phase two uses the projected reduction in the size of the poverty gap (i.e. a measure of how much the mean income of the poor falls below the poverty line) per dose of vaccine, to prevent economic and social deprivations. Phase three prioritizes countries with higher disease transmission rates. While building on published COVAX plans, these two models $[9,10]$ limit the range of factors considered to assess vaccine needs and fail to account for simultaneously overlapping objectives (health, social, and economic).

\section{A complementary framework: COVID-NEEDS}

We propose a framework (see Table 1) encompassing a broader range of health, social, and economic factors (Clinical vulnerability, Outbreak response systems, Virological features, Incidence and spread, Delivery and hesitancy, Net population susceptibility, Economic vulnerability, Economic power, Demand on health system, Social vulnerability (COVID-NEEDS)) to formulate a broad and standardized assessment of vaccine needs across countries. We selected each domain in the framework on the basis of 2020-2021 guidance on vaccine prioritization from public health agencies [11-14], evidence of impact of COVID-19 and associated non-pharmaceutical interventions $[15,16]$, and issues relating to the success of vaccination programmes $[17,18]$. We excluded factors if we did not consider them equally useful across different country contexts. Given that this is an evolving area, the framework is not without limitations, but facilitates a more in-depth analysis of needs for vaccines across countries, which can support the existing COVAX Facility needs assessment. We propose a list of sources from which to gather such information and 


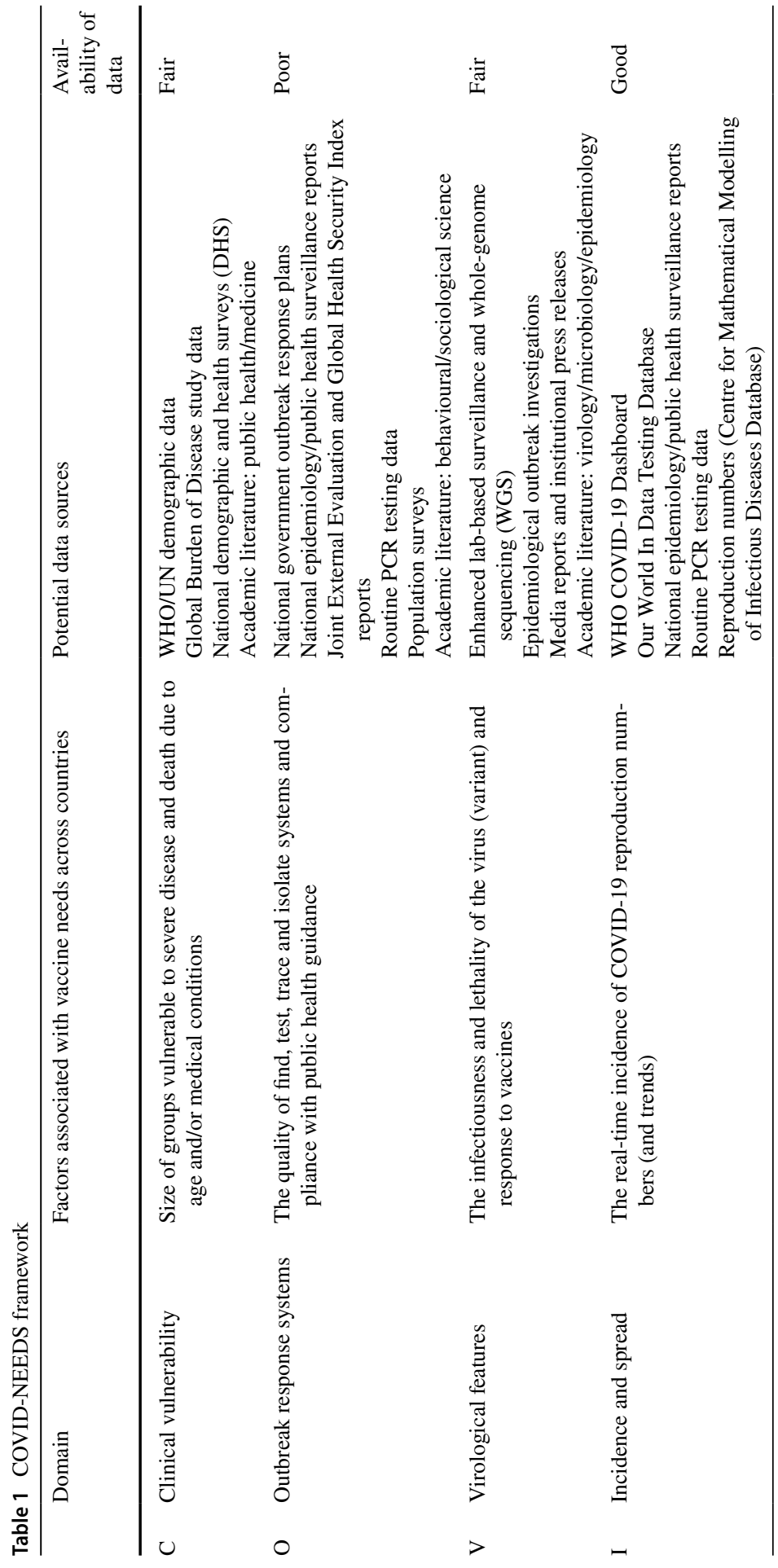

站。 


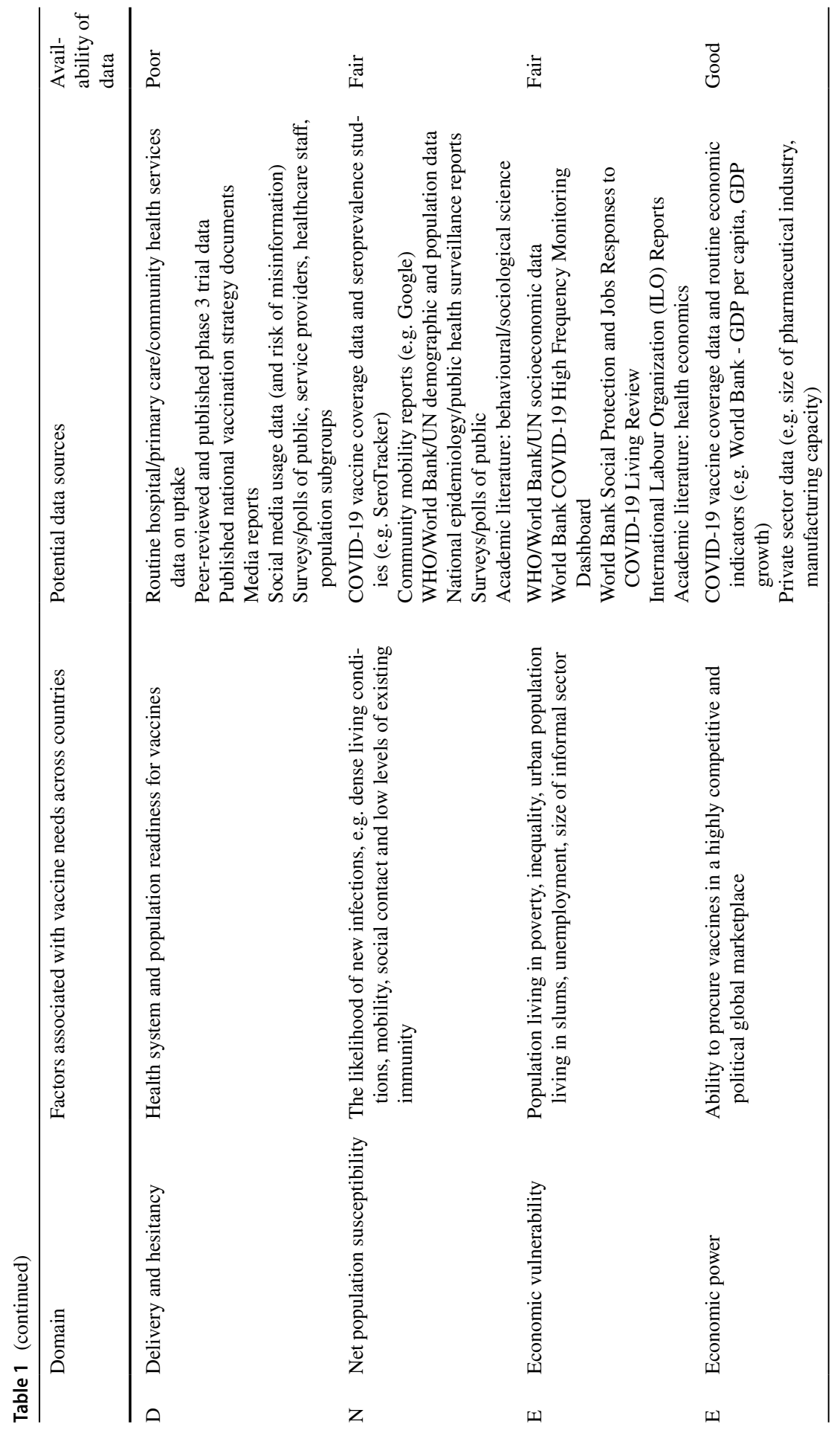




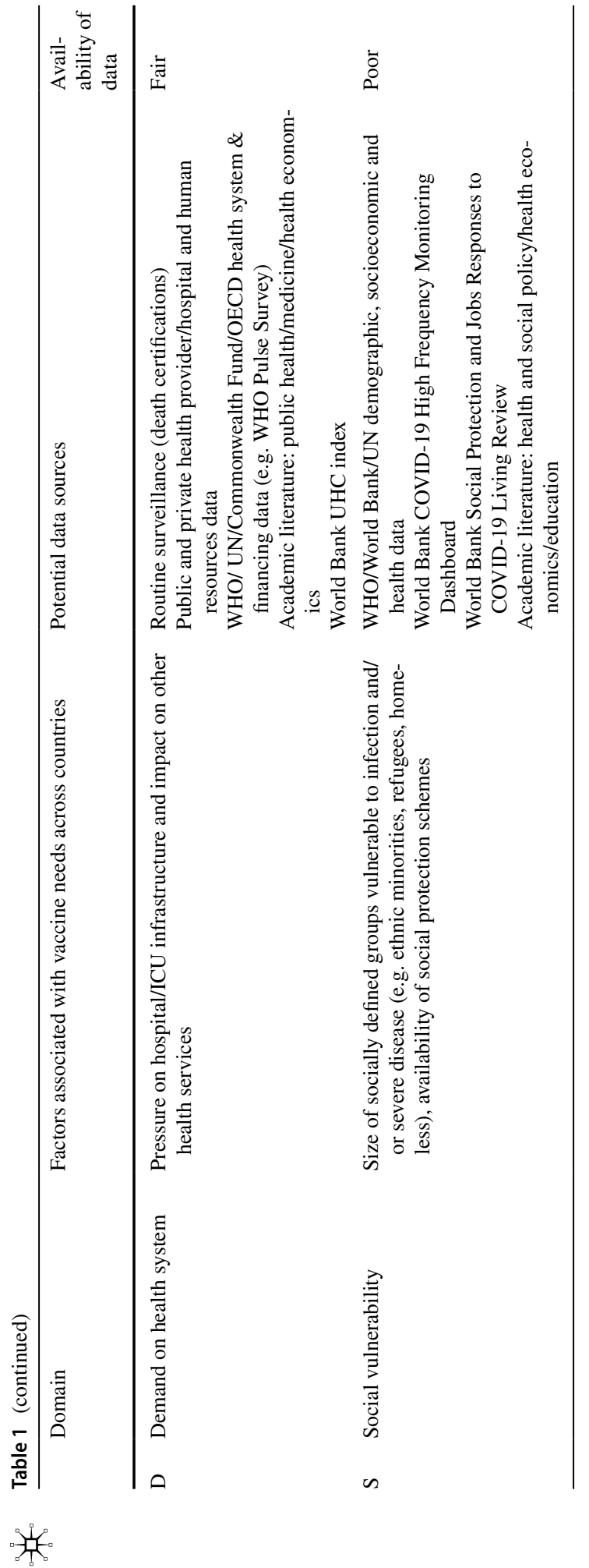


an assessment of the availability of international data (as good, fair or poor) for each COVID-NEEDS factor included. We considered data as 'good' if widely available for all countries through recent objective assessment from a single source, 'fair' if data exist at the international level but we found them to be of poor quality or missing for some countries, and 'poor' if the data do not exist in the public domain, at the international level.

The COVID-NEEDS framework as we propose here does not weight or rank the different domains. Depending on the core objectives of global and national vaccine programmes, some domains may be more important than others. Subsequent research will be needed to explore preferences and rankings, where researchers will ask public health experts and other stakeholders to weight each domain relative to others to define a prioritization score to aid decision-making. In the absence of a scoring tool, the framework is valuable because it makes explicit the many empirically grounded trade-offs involved in decisions on vaccine allocation.

\section{The factors}

Clinical vulnerability plays a key role in the severity of COVID-19, with the elderly being at highest risk [19]. High-income countries like Belgium, Italy, and the United Kingdom (UK), with relatively elderly populations, have experienced more deaths from COVID-19 (as a proportion of their populations) compared to countries with younger populations [20]. Medical conditions that increase vulnerability to COVID19, such as cardiovascular disease and diabetes, occur at younger ages and are less well controlled in LMICs [21]. High-risk conditions may not be homogenous across countries, with risk factors for COVID-19 severity like HIV and TB occurring more commonly in some countries than in others. Despite weak information systems in low-income countries, a 2020 descriptive study estimated the size of high-risk target groups across 194 WHO Member States, based on age and co-morbidities. Supported by longer-term efforts to improve disease surveillance and the accuracy of such estimates, COVAX should include such risk factors for COVID-19 severity when evaluating vaccine needs because distributions of age and health, and their associated levels of risk with COVID-19, vary greatly between countries.

Outbreak response systems are vital in infectious disease control, and much investment has gone into this during the pandemic. South Korean researchers cited the quality of such systems as key reasons for the very limited impacts of COVID-19 in South Korea, despite presence of a relatively old and therefore high-risk population [22]. Countries without effective contact tracing, testing, isolation and quarantine measures, or where the need for these interventions outstrip capacity, are likely to depend more on vaccines for disease control.

Virological features, related to mutations in the virus causing COVID-19, are an important and changing feature of relative vaccine need. Novel variants such as Delta, initially detected in India in late 2020, or the Gamma variant circulating in much of South America since early 2021, are affecting some countries more than others. In the UK, data show that the delta variant is not just more infectious, but also more lethal than the previous variant [23]. Some vaccines may be less effective 
against new variants. The South African government suspended the rollout of the Oxford-AstraZeneca vaccine in February 2021 due to concerns about the efficacy of the vaccine against the dominant Beta variant [24]. The likelihood of vaccination programme failure in one country compared to another, due to lower vaccine efficacy as well as increased viral transmission, or lethality, may further help to determine relative needs. Quantitative data on variants will be insufficient to operationalize virologic characteristics as criteria because country level data vary greatly. Thus, use of these criteria for prioritization should be supported by a qualitative assessment to not unfairly disadvantage those countries with less effective systems for genomic surveillance.

Delivery and hesitancy refer to the capacity of health systems to use allocated vaccines, and population readiness for vaccination. Population readiness is affected by perceived vulnerability to disease and the benefit of vaccination, public trust in government institutions and processes, as well as clear and timely communication of the scientific evidence underpinning vaccines [25]. For example, India rolled out the country's first indigenous vaccine, Covaxin, to healthcare workers early in 2021 before the publication of phase 3 trial results [26]. Consequently large numbers of health care workers hesitated, and uptake lagged compared to the OxfordAstraZeneca vaccine [27]. Some countries may have low levels of hesitancy but lack the health system readiness to quickly distribute and administer vaccines locally. The Fair Priority Model ignores this, as the authors acknowledge [10]. Distributing vaccines to countries where population hesitancy and health sector readiness have not been adequately addressed may result in wasted doses and inefficiency. For international decision-makers to know where vaccine allocation may have the most impact, it will be important to consider both hesitancy and readiness. To not unfairly penalize countries in need, multilateral or bilateral vaccine allocation mechanisms should not use these as exclusionary criteria but rather as indicators for further support to accompany vaccine delivery.

Incidence and spread are standardized and accessible metrics that can aid in national comparisons. Case numbers per 100,000 are available for all countries, and most also publish test positivity rates, accounting for differing testing capacities and providing a more comprehensive assessment of likely community transmission. Both the Fair Priority Model and the COVAX Facility propose to use metrics of transmissibility, or the effective reproductive numbers (i.e. the average number of secondary cases per infectious case in a population that includes both susceptible and non-susceptible individuals) as a measure of disease spread. Although this is more difficult to estimate accurately, $\mathrm{R}$ numbers provide more timely measures of disease transmission compared with estimates of case numbers. The latter offer limited value due to the long incubation period of COVID-19, the number of infected people with mild or absent symptoms, and limited PCR testing. Unlike other factors with more complex methods of measurement, monitoring trends in cases, test positivity, and transmissibility provide routine real-time information on the control of an epidemic. It takes time for the benefits of vaccines to be seen; meaning that real-time incidence and spread are relatively limited in utility. Despite the inclusion of the R number in current global vaccine needs assessment methods, it must form only part of a broader assessment of future vaccine needs. 
Net population susceptibility can be assessed through comparing levels of population immunity with contact-related risks of acquiring infection. Experts believe that high levels immunity could be responsible for declining rates of infection in the absence of non-pharmaceutical interventions in some countries [28, 29]. As vaccines are rolled out, populations will achieve varying levels of vaccine-derived immunity against COVID-19, based on how quickly vaccinators deliver and administer them, vaccine efficacy, and duration of immunity. Calculating a ratio to compare this estimated immunity with well-established risk factors for acquiring infection [30], including the size of dense, mobile, and socially active populations, can provide an understanding of net population susceptibility, informing the overall assessment of vaccine needs.

Economic vulnerability, particularly in terms of the impact of non-pharmaceutical interventions such as national or local lockdowns, is, for many, the defining feature of the pandemic. Lower resource countries and communities may be less able to institute non-pharmaceutical interventions due to economic costs and logistical constraints. Even high-income countries have suffered economically. In the UK, which has financially supported employers and workers using a furlough scheme, unemployment reached over 5\% of the working age population in September 2020, for the first time since 2015 [31]. Vaccines can protect jobs and livelihoods and prevent unemployment and rising levels of income inequality, which affect long-term health behaviours, services, and outcomes.

Economic Power has emerged as a key determinant of national access to vaccines. As of June 2021, high- and upper-middle-income countries, representing onefifth of the world's population of 7.8 billion people, bought approximately 6 billion doses of COVID-19 vaccines; while others, representing four-fifths of the population, secured only 2.6 billion [4]. To move towards an equitable distribution of vaccines, the global system must support those countries that would otherwise be unable to compete financially. As part of COVAX, the Advance Market Commitment (AMC) will support the purchase of vaccines for lower-income economies, under a different set of terms and costs compared to high-income (or self-financing) economies [11]. For global vaccine allocation to align with needs, the ability for countries to procure vaccines commercially (regardless of their needs relative to others) must be accounted for.

Demands placed on health systems are of paramount importance in preventing direct morbidity and mortality from COVID-19. Resource pressures from COVID19 also have indirect effects on other health services and health-seeking behaviours. A WHO survey of all countries revealed that almost every country (90\%) experienced some disruption, with greater disruptions reported in low- and middle-income than in high-income countries. The most frequently disrupted services included routine immunization [outreach services (70\%) and facility-based services (61\%)], noncommunicable disease diagnosis and treatment (69\%), family planning and contraception (68\%), treatment for mental health disorders $(61 \%)$, antenatal care $(56 \%)$, and cancer diagnosis and treatment (55\%). Given the centrality of COVID-19 health services and routine non-COVID-19 services in preventing morbidity and mortality, the assessment of national vaccine needs should include the real-time and predicted future capacity of health systems. 
Social vulnerability is the final domain in the framework. The distribution of COVID-19 disease burden and the impact of non-pharmaceutical interventions have exacerbated social inequalities. In the UK, those from ethnic minority backgrounds and those living in less well-resourced areas have been affected most adversely in terms of risk of disease acquisition and severity [34], Some population groups such as refugees, the homeless, and the incarcerated, have high risks of disease acquisition and severe disease or death [35]. In addition, there has been wide variation in social protection responses to the pandemic [36]. Some countries provide little or no social safety net to ensure food, housing, income, and healthcare during periods of COVID-19 restrictions. In populations with large socially or economically vulnerable groups, where social protection mechanisms are weak, COVID-19 vaccines may be more urgently needed to protect public health.

\section{Remaining challenges}

Data for some domains may be more readily available than for others due to limited data collection and reporting mechanisms in many LMICs. The available quantitative data (including its quality) must be considered alongside qualitative information from stakeholders within countries and familiar with real-time on-the-ground realities. More research will be required to better understand how to use these different sources of information together in a complementary way to best inform decisionmaking. Note that the current COVAX plans propose using both qualitative and quantitative data for the same purpose, therefore we anticipate that the process to operationalize the COVID-NEEDS framework will require few additional inputs beyond those already present in WHO processes.

We acknowledge that, as with any attempt to consolidate data on equitable vaccine allocation into a single model, populating this framework for different countries will be challenging. The collection and use of data has proliferated during the pandemic and proven fundamental for the assessment of and response to COVID19 outbreaks [38]. Data about most factors in the framework are already routinely collected and readily available. Using such frameworks could also incentivize the development of data collection and reporting mechanisms in some countries, with long-term benefits for wider public health objectives.

The use of such a framework will be limited by political buy-in and complexity. For domestic allocation of COVID-19 vaccines, countries such as the UK and the United States (US) have opted for relatively simple frameworks to increase the speed at which populations get immunized [39, 40]. For international allocation, speed, logistics, and acceptability have been similarly important, as reflected in COVAX plans. COVID-NEEDS is therefore not an off-the-shelf methodology to compare country needs, but a framework for informing discussions along with other methods such as those currently used by COVAX. It will be particularly valuable for expanding discussions to better consider needs across countries, which the COAX risk assessment has deemed to be similar. 


\section{Conclusions}

Estimating needs for COVID-19 vaccines is challenging. But given the broad impact of the pandemic across societies, and similarities in some traditional epidemiological metrics across countries, the narrow approaches currently in use have limitations. The COVID-NEEDS framework offers additional factors to supplement current methods and facilitate a more comprehensive assessment of vaccine needs across countries. The framework presents an opportunity to ensure that global decisionmakers prioritize vaccines in accordance with need, rather than desire, convenience, or ability to pay. This will be of interest for governments supplying other countries with vaccines through bilateral deals, as well as for international mechanisms such as COVAX, pursuing the equitable global allocation of COVID-19 vaccines. To weight factors in the framework and to evaluate its practical utility for better aligning global vaccine allocation with population needs, further research is needed. Given the threat of future pandemics, and the limited evidence for assessing public health needs for emergency countermeasures across countries, this framework may also inform plans for future global health emergency responses.

Author contributions Both VJ and PL were involved in the inception, design, research, data extraction, analysis, write-up, and editing of this manuscript. VJ was the lead author and PL acted as the supervisor for this paper.

Funding None required.

Data materials availability All data used and drawn upon are available in the manuscript, no additional data.

\section{Declarations}

Conflict of interest The authors declare that they have no conflict of interest.

\section{References}

1. Wellcome. When will the world be vaccinated against Covid-19? 2021; Available at: https://wellc ome.org/news/when-will-world-be-vaccinated-against-covid-19. Accessed 11 Mar 2021.

2. Our World in Data. Coronavirus (COVID-19) Cases. 2021; Available at: https://ourworldindata.org/ covid-cases. Accessed 01 June 2021.

3. Centre for Mathematical Modelling of Infectious Diseases. Epiforecasts: Global Summary. 2021; Available at: https://epiforecasts.io/covid/posts/global/. Accessed 19 June 2021.

4. World Health Organization. WHO Concept for fair access and equitable allocation of COVID-19 health products. 2020; Available at: file:///Users/Vageesh/Downloads/who-covid19-vaccine-allocation-final-working-version-9sept.pdf. Accessed 08 Feb 2021.

5. World Health Organization. Allocation Mechanism for COVAX Facility Vaccines. 2020; Available at: https://www.who.int/docs/default-source/coronaviruse/allocation-of-covax-f-vaccines-explainerv3-db.pdf?sfvrsn=516b3714_16. Accessed 14 Nov 2020.

6. World Health Organization. Access and allocation: how will there be fair and equitable allocation of limited supplies? 2021; Available at: https://www.who.int/news-room/feature-stories/detail/ 
access-and-allocation-how-will-there-be-fair-and-equitable-allocation-of-limited-supplies. Accessed 02 Feb 2021.

7. World Bank Group. Population ages 65 and above (\% of total population). 2019; Available at: https://data.worldbank.org/indicator/SP.POP.65UP.TO.ZS. Accessed 02 Aug 2021.

8. World Bank Group. Physicians (per 1000 people). 2018; Available at: https://data.worldbank.org/ indicator/SH.MED.PHYS.ZS. Accessed 02 Aug 2021.

9. Liu Y, Salwi S, Drolet BC. Multivalue ethical framework for fair global allocation of a COVID-19 vaccine. J Med Ethics. 2020;46(8):499-501.

10. Emanuel EJ, PersadD - Sturm College of Law, University of Denver, Denver, CO,,U.S.A., Kern A, Buchanan A, Fabre C, Halliday D, et al. An ethical framework for global vaccine allocation. Science. 2020;369(6509):1309-12.

11. Gavi. The Gavi COVAX AMC Explained. 2020; Available at: https://www.gavi.org/vaccineswo rk/gavi-covax-amc-explained\#: :text=The\%20Gavi\%20COVAX\%20Advance $\% 20$ Market,access $\%$ 20to\%20COVID\%2D19\%20vaccines. Accessed 18 June 2021.

12. World Health Organization. WHO SAGE values framework for the allocation and prioritization of COVID-19 vaccination. 2020; Available at: https://apps.who.int/iris/bitstream/handle/10665/ 334299/WHO-2019-nCoV-SAGE_Framework-Allocation_and_prioritization-2020.1-eng.pdf? ua=1. Accessed 03 Feb 2021.

13. European Centre for Disease Prevention and Control (ECDC). COVID-19 vaccination and prioritisation strategies in the EU/EEA. 2020; Available at: https://www.ecdc.europa.eu/en/publicationsdata/covid-19-vaccination-and-prioritisation-strategies-eueea. Accessed 03 Feb 2021.

14. Centers for Disease Control and Prevention (CDC). CDC's COVID-19 Vaccine Rollout Recommendations. 2021; Available at: https://www.cdc.gov/coronavirus/2019-ncov/vaccines/recommenda tions.html. Accessed 03 Feb 2021.

15. World Bank Group. COVID-19 High-Frequency Monitoring Dashboard. 2020; Available at: https:// www.worldbank.org/en/data/interactive/2020/11/11/covid-19-high-frequency-monitoring-dashb oard. Accessed 03 Feb 2021.

16 Cheng C, Barceló J, Hartnett AS, Kubinec R, Messerschmidt L. COVID-19 government response event dataset (CoronaNet v. 1.0). Nat Hum Behav. 2020;4(7):756-68.

17. Mahase E. Covid-19: What new variants are emerging and how are they being investigated? BMJ. 2021;372:n158.

18. Wang W, Wu Q, Yang J, Dong K, Chen X, Bai X, et al. Global, regional, and national estimates of target population sizes for covid-19 vaccination: descriptive study. BMJ. 2020;371:m4704.

19. Williamson E, Walker AJ, Bhaskaran KJ, Bacon S, Bates C, Morton CE, et al. OpenSAFELY: factors associated with COVID-19-related hospital death in the linked electronic health records of 17 million adult NHS patients. medRxiv 2020.

20. Statista. Coronavirus (COVID-19) deaths worldwide per one million population as of January 27 , 2021, by country. 2021; Available at: https://www.statista.com/statistics/1104709/coronavirusdeaths-worldwide-per-million-inhabitants/. Accessed 27 Jan 2021.

21. World Bank Group. Noncommunicable Disease Risk Factors in Developing Countries: Policy Perspectives. 2017; Available at: https://openknowledge.worldbank.org/handle/10986/29359. Accessed 28 Jan 2021.

22. Park YJ, Choe YJ, Park O, Park SY, Kim YM, Kim J, et al. Contact tracing during coronavirus disease outbreak, South Korea, 2020. Emerg Infect Dis. 2020;26(10):2465-8.

23. Public Health England. New COVID-19 (SARS-CoV-2) variants. 2021; Available at: https://www. gov.uk/government/collections/new-sars-cov-2-variant. Accessed 08 Feb 2021.

24. BBC. Covid: South Africa halts AstraZeneca vaccine rollout over new variant. 2021; Available at: https://www.bbc.co.uk/news/world-africa-55975052. Accessed 08 Feb 2021.

25. DELVE Initiative. SARS-CoV-2 Vaccine Development \& Implementation; Scenarios, Options, Key Decisions. The Royal Society 2020.

26. The Wire. Despite Mounting Disputes, Govt Presses Ahead With Vaccine Roll Out. 2021; Available at: https://science.thewire.in/health/covaxin-covishield-indian-government-rollout-peoples-hospitalphase-3-clinical-trials-centralisation-pm-cares/. Accessed 27 Jan 2021.

27. The Times of India. Rajasthan: $68.42 \%$ turnout on Day 4 of vaccination drive. 2021; Available at: https://timesofindia.indiatimes.com/city/jaipur/68-42-turnout-on-day-4-of-vaccination-drive/artic leshow/80412767.cms. Accessed 27 Jan 2021.

28. The Economic Times. Localised herd immunity, young population behind the dip in India's Covid count? 2021; Available at: https://health.economictimes.indiatimes.com/news/diagnostics/local 
ised-herd-immunity-young-population-behind-the-dip-in-indias-covid-count/80110827. Accessed 27 Jan 2021.

29 Poustchi H, Darvishian M, Mohammadi Z, Shayanrad A, Delavari A, Bahadorimonfared A, et al. SARS-CoV-2 antibody seroprevalence in the general population and high-risk occupational groups across 18 cities in Iran: a population-based cross-sectional study. Lancet Infect Dis. 2020;21(4):473-81.

30. Public Health England. Factors contributing to risk of SARS-CoV2 transmission associated with various settings. 2020; Available at: https://assets.publishing.service.gov.uk/government/uploa ds/system/uploads/attachment_data/file/945978/S0921_Factors_contributing_to_risk_of_SARS_ 18122020.pdf. Accessed 08 Feb 2021.

31. Office for National Statistics. Unemployment. 2021; Available at: https://www.ons.gov.uk/emplo ymentandlabourmarket/peoplenotinwork/unemployment. Accessed 08 Feb 2021.

32. The New York Times. See How the Vaccine Rollout Is Going in Your State. 2021; Available at: https://www.nytimes.com/interactive/2020/us/covid-19-vaccine-doses.html. Accessed 2 Feb 2021.

33. World Health Organization. Pulse survey on continuity of essential health services during the COVID-19 pandemic: interim report, 27 August 2020. 2020; Available at: https://www.who.int/ publications/i/item/WHO-2019-nCoV-EHS_continuity-survey-2020.1 Accessed 08/09, 2021.

34. Public Health England. Disparities in the risk and outcomes of COVID-19. 2020; Available at: https://assets.publishing.service.gov.uk/government/uploads/system/uploads/attachment_data/file/ 908434/Disparities_in_the_risk_and_outcomes_of_COVID_August_2020_update.pdf. Accessed 26 Jan 2021.

35. The Health Foundation. Emerging evidence on health inequalities and COVID-19: March 2021. 2021; https://www.health.org.uk/news-and-comment/blogs/emerging-evidence-on-health-inequaliti es-and-covid-19-march-2021. Accessed 17 June 2021.

36. Gentilini U, Almenfi M, Orton I, Dale P. Social protection and jobs responses to COVID-19. 2020.

37. UNESCO. Duration of complete and partial school closures. 2021; Available at: https://en.unesco. org/news/unesco-figures-show-two-thirds-academic-year-lost-average-worldwide-due-covid-19school. Accessed 28 Jan 2021.

38 Whitelaw S, Mamas MA, Topol E, Van Spall HG. Applications of digital technology in COVID19 pandemic planning and response. Lancet Digit Health. 2020. https://doi.org/10.1016/S25897500(20)30142-4.

39. Department of Health and Social Care. JCVI: updated interim advice on priority groups for COVID19 vaccination. 2020; Available at: https://www.gov.uk/government/publications/priority-groupsfor-coronavirus-covid-19-vaccination-advice-from-the-jcvi-25-september-2020/jcvi-updated-inter im-advice-on-priority-groups-for-covid-19-vaccination. Accessed 18 Nov 2020.

40. Jain V, Schwarz L, Lorgelly P. A rapid review of COVID-19 vaccine prioritization in the US: alignment between Federal guidance and State practice. Int J Environ Res Public Health. 2021;18(7):3483.

Publisher's Note Springer Nature remains neutral with regard to jurisdictional claims in published maps and institutional affiliations.

Vageesh Jain MBBS MPH, Academic Clinical Fellow in Public Health \& ST4 Public Health Registrar, Institute for Global Health, University College London, London, UK

Paula Lorgelly Bsc (Hons), PhD PGCAP, Professor of Health Economics, Department of Applied Health Research, Institute of Epidemiology \& Health Care, University College London, London, UK 